\title{
Energy Saving of Base Stations Sleep Scheduling for Multi-Hop Vehicular Networks
}

\author{
Tao Han*, Xiong Liu*, Lijun Wang ${ }^{\dagger}$, Jiang Wang ${ }^{\ddagger}$, Kyung Sup Kwak ${ }^{\S}$ and Qiang $\mathrm{Li}^{*}$ \\ *School of Electronic Information and Communications, Huazhong University of Science and Technology, Wuhan, China \\ ${ }^{\dagger}$ Department of Information Science and Technology, Wenhua College, Wuhan, China \\ ${ }^{\ddagger}$ Shanghai Research Center for Wireless Communication, Shanghai, China \\ ${ }^{\ddagger}$ Shanghai Institute of Microsystem and Information Technology, Chinese Academy of Sciences, Shanghai, China \\ $\S$ Inha Hanlim Fellow Professor, Department of Information and Communication, Inha university, Incheon, Korea \\ Email: * ${ }^{*}$ hantao, m201571774, qli_patrick $\} @$ hust.edu.cn, ${ }^{\dagger}$ wanglj22@163.com, ${ }^{\dagger}$ jiang.wang@wico.sh, ${ }^{\S}$ kskwak@inha.ac.kr
}

\begin{abstract}
This paper investigates the energy saving of base station (BS) deployed in a 1-D multi-hop vehicular network with sleep scheduling strategy. We consider cooperative BS scheduling strategy where BSs can switch between sleep and active modes to reduce the average energy consumption utilizing the information of vehicular speeds and locations. Assuming a Poisson distribution of vehicles, we derive an appropriate probability distribution function of distance between two adjacent cluster heads, where a cluster is a maximal set of vehicles in which every two adjacent vehicles can communicate directly when their Euclidean distance is less than or equal to a threshold, known as the communication range of vehicles. Furthermore, the expected value of the sojourn time in the sleep mode and energy saving are obtained. The numerical results show that the sleep scheduling strategy significantly reduces the energy consumption of the base stations
\end{abstract}

Index Terms-Energy saving; sleeping strategy; vehicular networks

\section{INTRODUCTION}

Vehicular ad hoc network (VANET) is a mobile multi-hop network formed by vehicles traveling on the road and utilizes multi-hop communication to improve connectivity probability of every vehicle. In conventional infrastructure-based vehicular communication networks, information are transmitted to every vehicle by base stations (BSs) deployed alongside the road. While in VANETs, two vehicles can communicate with one another directly as long as the Euclidean distance between two vehicles is less than a threshold, the communication range of a vehicle, defined as $r_{0}$ in this paper [1]. Due to the mobility of vehicles, the topology of a VANET is changing over time [2],

\footnotetext{
${ }^{1}$ The corresponding author is Lijun Wang. The authors would like to acknowledge the support from the International Science and Technology Cooperation Program of China (Grant No. 2015DFG12580 and 2014DFA11640), the National Natural Science Foundation of China (NSFC) (Grant No. 61471180, 61301128, 61461136004, and 61271224), the NSFC Major International Joint Research Project (Grant No. 61210002), Hubei Provincial Department of Education Scientific research projects (Grant No. B2015188), Shanghai Action plan of science and technology innovation (Grant No. 15511103200), the Fundamental Research Funds for the Central Universities (HUST Grant No. 2015MS038), the Special Research Fund for the Doctoral Program of Higher Education (Grant No. 20130142120044), Wenhua College (Grant No. 2013Y08), and EU FP7-PEOPLE-IRSES (Contract/Grant No. 247083, 318992 and 610524). Kyung Sup Kwak's work is supported by National Research Foundation of Korea-Grant funded by the Korean Government (Ministry of Science, ICT and Future Planning-NRF-2014K1A3A1A20034987).
}

so the clusters of vehicles are splitting and merging over time, where a cluster is a maximal set of vehicles in which there is at least one hop between each pair of vehicles. Therefore, the information propagation is distinct from conventional wireless networks.

The ever increasing data demand of vehicles leads to more operation power of BSs, assumed as the linear function of transmission power, in infrastructure-based vehicular networks [3]. Especially when there is no vehicle on the road, e.g., at night, BS's operation will waste lots of energy. A useful solution is to turn off the BS if no vehicle is in its range [4]. The dynamic network topology makes it difficult to design a mechanism of BS sleeping schedule [5], especially when multi-hop communication among vehicles are considered. Multi-hop communication allows a BS only need to communicate to only one vehicle of a cluster, then by vehicle to vehicle communication, all vehicles in a cluster can receive or transmit messages.

In this paper, our main contributions are as follows: 1) we develop a statistic energy saving model for 1-D multi-hop vehicular network with BSs uniformly deployed at roadside; 2) the probability distribution function (PDF) for the distance between two adjacent cluster heads is provided; 3) the expected value of the sojourn time in the sleep mode and the expected energy saving of a BS are obtained; 4) the impact of network parameters on energy saving is investigated.

The rest of this paper is organized as follows: Section II reviews related work. Section III describes the system model. The analysis on the energy efficiency is presented in Section IV. Section V discusses the impacts of network parameters on energy saving. Finally Section VI concludes this paper and proposes future work.

\section{RELATED WORK}

In recent years, VANETs have attracted significant interest due to their large number of potential applications. Most of them consider the connectivity of vehicles or algorithm for cluster formation, the authors of [6] took vehicle to infrastructure communication and vehicle to vehicle communication into consideration, connectivity probability of platoonbased VANETs where vehicles follow a Poisson distribution 
is derived, and they investigated the relationship between connectivity probability and network parameters. In [7], Zhang et al. considered a model in which time is divided into time slots of equal length and each vehicle changes its speed at the beginning of each time slot, independent of its speed in other time slots. They derived a closed form expression for the PDF of cluster length using queue theory. The authors of [8] investigated the optimum deployment of road side units to maximize connectivity of vehicular networks. As for BS sleeping algorithm, there are a lot of research in this field. [9] proposed a switch on/off algorithm for BSs, which exploits the knowledge of the distance between the user equipments and their associated BSs in the LTE-Advanced cellular networks. They obtained the relation between energy consumption and time of day. In [10], Lee et al. proposed an energy efficient power control mechanism for BS in mobile communication system in order to improve efficient use of BS transmission power. BSs monitor the number of nodes in sector and controls transmission power accordingly. In [11], Han et al. investigated the energy efficiency of BS sleep scheduling strategies in 1$\mathrm{D}$ infrastructure-based vehicular networks where vehicles are Poissonly distributed and the vehicle speeds are uniformly distributed. BSs can schedule their sleep by utilizing the information of vehicles' location and speed. Analytical results on the expected amount of energy saving of a BSs are derived, which is shown to be a function of vehicular density and speed distribution. The study in [11] did not consider vehicle-tovehicle communication which will greatly reduce the energy consumption as illustrated in this paper afterwards.

\section{System MODEL}

This paper considers a vehicular network employing both vehicle to vehicle communication and vehicle to infrastructure communication. The spatial distribution of vehicles follows a homogeneous Poisson process with density $\rho$ [12]. In this paper, the speed of each vehicle is considered to be independently and identically distributed following a common uniform distribution [13]. Specifically, the PDF of vehicular speed denoted by $V$ is

$$
f_{v}(v)=\left\{\begin{array}{ll}
\frac{1}{b-a}, & a<v<b \\
0, & \text { otherwise }
\end{array},\right.
$$

where $b$ and $a$ represent the maximal and minimal speed, respectively, and we only consider vehicles traveling in one direction. As commonly done in this field, we assume that vehicular speed remains constant over time to simplify the analysis.

A set of BSs are regularly deployed with distance $D$ between adjacent BSs, as illustrated in Fig. 1. Typical values of $D$ depends on practical implementations [14]. The communication range of a $\mathrm{BS}$ is $D$ with $\mathrm{BS}$ as the center.

In this paper, we define the first vehicle in the direction of travel of a cluster as the cluster head $(\mathrm{CH})$, only the $\mathrm{CH}$ can directly communicate with its nearest BS and other vehicles in the cluster must communicate with $\mathrm{BS}$ via the $\mathrm{CH}$ by multihop communication.

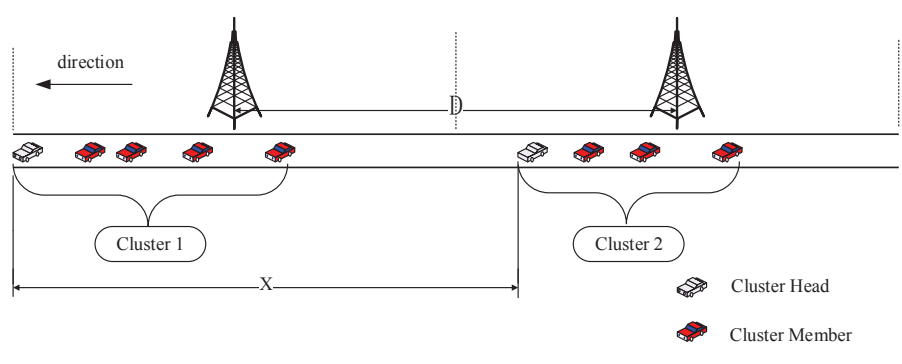

Fig. 1. System Model

Further, it is assumed that when a $\mathrm{CH}$ arrives or leaves the range of a $\mathrm{BS}$, the $\mathrm{BS}$ can detect it by listening to the indicating signal sent from the $\mathrm{CH}$. A BS can be in either an active mode or a sleep mode. In the active mode, a BS operates as normal; while in the sleep mode, a BS switches off its transmitter and amplifier to save energy. Therefore, a BS cannot transmit signal but can receive signal only for detecting $\mathrm{CHs}$ in its communication range when it is in the sleep mode. This paper considers the sleep scheduling strategy that BSs switch between the active mode and the sleep mode without affecting the QoS such as connectivity, a BS will keep active as long as there is a $\mathrm{CH}$ in its coverage.

We next describe the energy consumption model. Let $P_{\mathrm{a}}$ be the power consumed in the active mode which includes air conditioning, signal processing, power supply and power amplifier, etc. and let $P_{\mathrm{s}}$ be the power of signal processing unit for transmitting pilot signals when a BS in a sleep mode. [15]. So when a BS is in the sleep mode, the power saved is $P_{0}=P_{\mathrm{a}}-P_{\mathrm{s}}$. Further, consider that the energy cost of turning a BS off and then back on is $E_{\mathrm{c} 1}$ and $E_{\mathrm{c} 2}$ respectively. Then the total energy cost of turning a BS off and later back on is $E_{\mathrm{c}}=E_{\mathrm{c} 1}+E_{\mathrm{c} 2}$. This extra energy cost can have a impact on the energy saving achieved by a BS.

\section{AnAlysis OF ENERGY SAVING}

In this paper, we assume that vehicles are distributed following a Poisson distribution. Denote by $Y$ the Euclidean distance between two randomly chosen adjacent vehicles and $X$ the Euclidean distance between two randomly chosen adjacent $\mathrm{CHs}$ at a arbitrary time instant. It follows from the Poisson distribution of vehicle that the variable $Y$ follows an exponential distribution with parameter $\rho$. We define a variable $T$ as $T=\frac{X-D}{V}$ and $f_{t}(t)$ is its PDF. When $X$ is larger than $D, T$ represents the time period from the first cluster head leaves the communication range of a BS to the next cluster head enters the communication range of the BS. Then the BS can detect these events so that its transmitter and amplifier can be switched off to save energy and switched on afterwards, $T$ is the time period of BS in sleep mode. So the PDF of variable $X$ is the key point to work out the energy saved by our model. 


\section{A. Distribution of the distance between adjacent $\mathrm{CHs}$}

According to [7], the PDF of the cluster length $x_{0}$ is

$$
\begin{aligned}
f_{x_{0}}\left(x_{0}\right)= & \frac{\rho}{e^{\rho r_{0}}-1} \sum_{m=0}^{\left\lfloor x_{0} / r_{0}\right\rfloor} \frac{\left(-\rho\left(x_{0}-m r_{0}\right)\right)^{m-1}}{-m !} \\
& \times\left(\rho\left(x_{0}-m r_{0}\right)+m\right) e^{-\rho m r_{0}},
\end{aligned}
$$

the $\mathrm{CDF}$ of the distance between two adjacent $\mathrm{CHs} X$ is the sum of cluster length $x_{0}$ and a random truncated exponential variable $x_{1}$, the distance between the last vehicle of the cluster and the $\mathrm{CH}$ of the next cluster, whose PDF is

$$
f_{x_{1}}\left(x_{1}\right)=\left\{\begin{array}{ll}
\frac{\rho e^{-\rho x_{1}}}{e^{-\rho r_{0}}}, & x_{1}>r_{0} \\
0, & \text { otherwise }
\end{array},\right.
$$

so the CDF of $X$ can be calculated as

$$
\begin{aligned}
F_{x}(x) & =\int_{0}^{x-r_{0}} \int_{r_{0}}^{x-x_{0}} f_{x_{0}}\left(x_{0}\right) \cdot f_{x_{1}}\left(x_{1}\right) \mathrm{d} x_{1} \mathrm{~d} x_{0} \\
& =\int_{0}^{x-r_{0}} f_{x_{0}}\left(x_{0}\right) \mathrm{d} x_{0} \int_{r_{0}}^{x-x_{0}} \frac{\rho e^{-\rho x_{1}}}{e^{-\rho r_{0}}} \mathrm{~d} x_{1} \\
& =\int_{0}^{x-r_{0}} f_{x_{0}}\left(x_{0}\right)\left(1-e^{-\rho\left(x-x_{0}-r_{0}\right)}\right) \mathrm{d} x_{0} .
\end{aligned}
$$

Therefore, the PDF of $X$ follows that

$$
\begin{aligned}
f_{x}(x) & =\left[\int_{0}^{x-r_{0}} f_{x_{0}}\left(x_{0}\right)\left(1-e^{-\rho\left(x-x_{0}-r_{0}\right)}\right) \mathrm{d} x_{0}\right]^{\prime} \\
& =\left[\int_{0}^{x-r_{0}} f_{x_{0}}\left(x_{0}\right)-e^{-\rho\left(x-r_{0}\right)} e^{\rho x_{0}} f_{x_{0}}\left(x_{0}\right) \mathrm{d} x_{0}\right]^{\prime} \\
& =\rho e^{-\rho\left(x-r_{0}\right)} \int_{0}^{x-r_{0}} e^{\rho x_{0}} f_{x_{0}}\left(x_{0}\right) \mathrm{d} x_{0} .
\end{aligned}
$$

Substitute the preceding equation (2) into (5), because $f_{x_{0}}\left(x_{0}\right)$ is a piece-wise function, to calculate the integral, we need to divide the $x_{0}$ into segments of equal length $r_{0}$, and define $y=x_{0}-m r_{0}$, when $x \geqslant 2 r_{0}$, then we can obtain

$$
\begin{aligned}
f_{x}(x)= & \frac{-\rho e^{-\rho\left(x-r_{0}\right)}}{e^{\rho r_{0}}-1}\left\{\sum_{k=0}^{\left\lfloor x / r_{0}-1\right\rfloor} \sum_{m=0}^{k-1}\right. \\
& \int_{\rho(k-1-m) r_{0}}^{-\rho(k-m) r_{0}} \frac{y^{m}}{m !} e^{-y}-\frac{y^{m-1}}{(m-1) !} e^{-y} \mathrm{~d} y \\
& \left.+\int_{-\rho\left(\left\lfloor x / r_{0}-1\right\rfloor-m\right) r_{0}}^{-\rho\left(x-m r_{0}-r_{0}\right)} \frac{y^{m}}{m !} e^{-y}-\frac{y^{m-1}}{(m-1) !} e^{-y} \mathrm{~d} y\right\} \\
= & \frac{\rho e^{-\rho\left(x-r_{0}\right)} e^{\left\lfloor/ r_{0}-1\right\rfloor} k-1}{e^{\rho r_{0}-1}} \sum_{k=0}^{k-1}\left\{\sum_{m=0}\right. \\
& \frac{e^{\rho(k-m) r_{0}}\left[-\rho(k-m) r_{0}\right]^{m}}{m !} \\
& \left.-\frac{e^{\rho(k-m-1) r_{0}}\left[-\rho(k-m-1) r_{0}\right]^{m}}{m !}\right\} \\
& +\left\{\frac{e^{\rho\left(x-k r_{0}-r_{0}\right)}\left[-\rho\left(x-k r_{0}-r_{0}\right)\right]^{k}}{k !}\right. \\
& \left.-\frac{e^{\rho\left(\left\lfloor x / r_{0}-1\right\rfloor-k r_{0}\right)}\left[-\rho\left(\left\lfloor x / r_{0}-1\right\rfloor-k r_{0}\right)\right]^{k}}{k !}\right\},(6)
\end{aligned}
$$

when $r_{0} \leqslant x<2 r_{0}$,

$$
f_{x}(x)=\frac{\rho\left[1-e^{-\rho\left(x-r_{0}\right)}\right]}{e^{\rho r_{0}}-1} .
$$

According to the definition of mathematics expectation, we have

$$
\mathbb{E}[X]=\int_{r_{0}}^{\infty} x f_{x}(x) \mathrm{d} x .
$$

\section{B. Time interval of sleep mode}

As mentioned above, CHs transmit signals to BS if they come in or step off the coverage of the BS, so when a $\mathrm{CH}$ leaves the coverage of a $\mathrm{BS}$ and the next $\mathrm{CH}$ is not in its coverage, BS can turn off and then keep in sleep mode until the next $\mathrm{CH}$ comes in its coverage.

Thus the time interval of sleep mode for a typical BS can be defined as

$$
T_{\mathrm{off}}=\left\{\begin{array}{ll}
T, & T>0 \\
0, & T \leq 0
\end{array} .\right.
$$

Apparently, the PDF of $T_{\text {off }}$ is hard to obtain, so we may pay attention to its expected value which can be derived as

$$
\begin{aligned}
\mathbb{E}\left[T_{\text {off }}\right] & =\frac{\int_{0}^{\infty} t f_{t}(t) \mathrm{d} t}{\mathbb{P}\{T>0\}} \\
& =\frac{\int_{D}^{\infty} \int_{a}^{b} \frac{x-D}{v} \cdot f_{x}(x) \cdot f_{v}(v) \mathrm{d} v \mathrm{~d} x}{\mathbb{P}\{X>D\}} \\
& =\frac{\ln b-\ln a}{b-a} \cdot \frac{\int_{D}^{\infty}(x-D) f_{x}(x) \mathrm{d} x}{\int_{D}^{\infty} f_{x}(x) \mathrm{d} x} .
\end{aligned}
$$

In the following section, we will investigate the relation between $T_{\text {off }}$ and the system parameters.

\section{Average power saved}

In the same way, the active time period of a BS is denoted as $T_{\mathrm{on}}$, which is

$$
T_{\text {on }}=\left\{\begin{array}{ll}
\frac{D}{V}, & X>D \\
\frac{X}{V}, & X \leq D
\end{array} .\right.
$$

The energy saved $E_{\text {off }}$ in sleep mode can be valued by

$$
E_{\text {off }}=T_{\text {off }} P_{0}-E_{\mathrm{c}} .
$$

We denote $P_{\text {save }}$ as the power saved by our sleeping scheduling which can be calculated as

$$
P_{\text {save }}=\frac{T_{\text {off }} P_{0}-E_{\mathrm{c}}}{T_{\text {off }}+T_{\text {on }}},
$$

substitute the preceding equation (9) and (11) into (13), we get

$$
P_{\text {save }}=\left\{\begin{array}{ll}
\frac{\left(\frac{X-D}{V}\right) P_{0}-E_{\mathrm{c}}}{X / V}, & X>D \\
0, & X \leq D
\end{array} .\right.
$$


Then the expected value of the power saved for a typical BS denoted as $\mathbb{E}\left[P_{\text {save }}\right]$ can be calculated by

$$
\begin{aligned}
\mathbb{E}\left[P_{\text {save }}\right] & =\mathbb{E}\left[\frac{\frac{X-D}{V} \cdot P_{0}-E_{\mathrm{c}}}{X / V}\right] \cdot \mathbb{P}\{X>D\} \\
& =\mathbb{E}\left[\frac{(X-D) P_{0}-E_{\mathrm{c}} \cdot V}{X}\right] \cdot \mathbb{P}\{X>D\} \\
& =\mathbb{E}\left[P_{0}-\frac{D P_{0}}{X}-E_{\mathrm{c}} \frac{V}{X}\right] \cdot \mathbb{P}\{X>D\} .
\end{aligned}
$$

Due to the independence of $X$ and $D$, we have

$$
\mathbb{E}\left[\frac{V}{X}\right]=\mathbb{E}\left[\frac{1}{X}\right] \cdot \mathbb{E}[V],
$$

where

$$
\begin{gathered}
\mathbb{E}\left[\frac{1}{X}\right]=\frac{\int_{D}^{\infty} \frac{1}{x} f_{x}(x) \mathrm{d} x}{\mathbb{P}\{X>D\}}, \\
\mathbb{E}[V]=\frac{a+b}{2},
\end{gathered}
$$

and

$$
\mathbb{P}\{X>D\}=\int_{D}^{\infty} f_{x}(x) \mathrm{d} x=1-F(D) .
$$

Through the given results, we can obtain that

$$
\begin{aligned}
\mathbb{E}\left[P_{\text {save }}\right]= & P_{0} \cdot[1-F(D)]-P_{0} \cdot D \cdot \int_{D}^{\infty} \frac{1}{x} \cdot f_{x}(x) \mathrm{d} x \\
& -E_{\mathrm{c}} \cdot \mathbb{E}[V] \cdot \int_{D}^{\infty} \frac{1}{x} \cdot f_{x}(x) \mathrm{d} x .
\end{aligned}
$$

According to (20), we know the $\mathbb{E}\left[P_{\text {save }}\right]$ is related to the vehicular average speed and communication range $r_{0}$. The next section will show the relationship between the energy saving and these system parameters.

\section{Numerical Results}

This section shows numerical evaluation of the results presented in the previous sections, followed by discussions and conclusions. The typical values of system parameters are selected from certain practical scenarios. Specifically, the distance between BSs is $D=800 \mathrm{~m}$. The energy consumption model uses parameters $P_{0}=1 \mathrm{kw}$ and $E_{\mathrm{c}}=10 \mathrm{~J}$.

First, the expected value of the distance between two adjacent $\mathrm{CHs}$ shown in Fig. 2 is related to vehicular density and communication range. Undoubtedly, the larger $r_{0}$ means that vehicles have more opportunity to directly communicate with adjacent vehicles, so leading to more vehicles in a cluster and then larger value of $\mathbb{E}[X]$. And for a certain $r_{0}$, the value of $\mathbb{E}[X]$ decreases first and then increases to infinite as the vehicular density grows. In the case of very low vehicular density, the distance between two adjacent vehicles seems to be large enough and they cannot communicate directly, then the distance of two adjacent $\mathrm{CHs}$ is very large. With the increase of vehicular density, the distance between two adjacent vehicles decrease and some of them can communicate directly, so the value of $\mathbb{E}[X]$ may decrease, but if the vehicular density continuously increase, the distance of two adjacent vehicles all become less than $r_{0}$, causing a large amount of

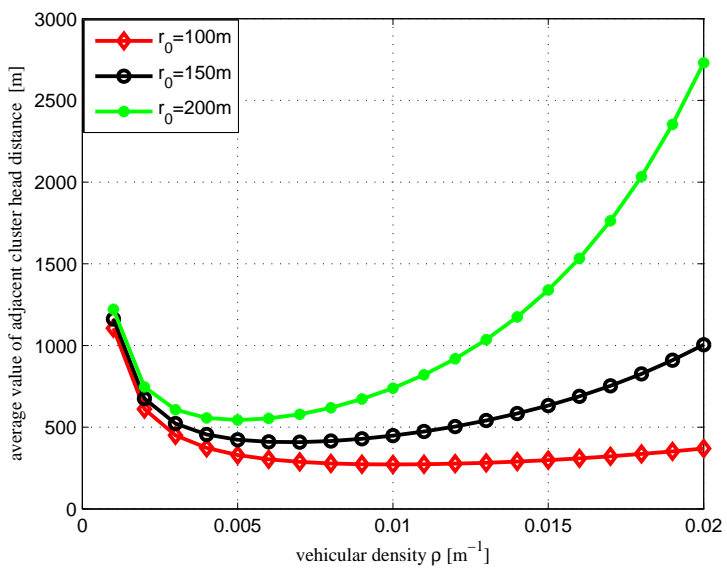

Fig. 2. The expected value of distance between two adjacent $\mathrm{CHs}$

vehicles in a cluster, leading to the increase of the value of $\mathbb{E}[X]$.

Then we investigate the relationship between the sojourn time of sleep mode and traffic parameters, such as vehicular density $\rho$ and transmission range $r_{0}$. Since the sojourn time in sleep model is derived from the $\mathbb{E}[X]$, the trend of $\mathbb{E}\left[T_{\text {off }}\right]$ is the same with $\mathbb{E}[X]$, which is clearly depicted in Fig. 3.

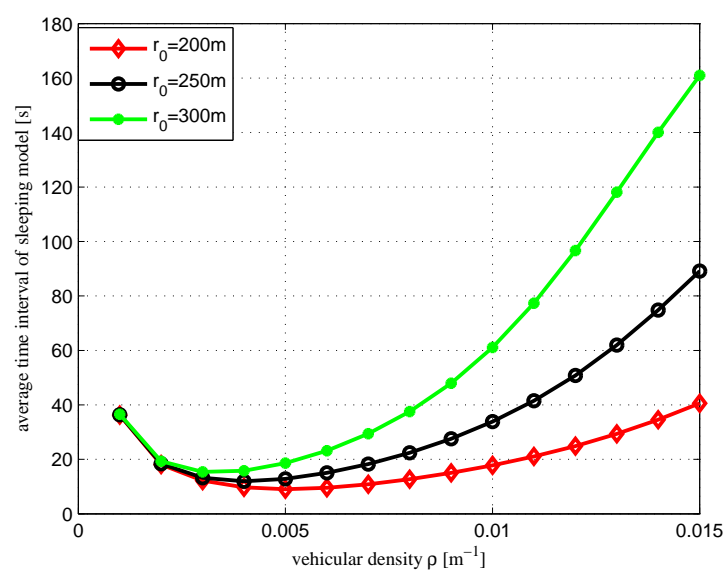

Fig. 3. Time interval of sleep mode versus vehicular transmission range, where $a=40 \mathrm{~km} / \mathrm{h}, b=80 \mathrm{~km} / \mathrm{h}$.

Next we come to the average power saved $\mathbb{E}\left[P_{\text {save }}\right]$, the relationship between $\mathbb{E}\left[P_{\text {save }}\right]$ and vehicular density is clearly presented in Fig. 4. The larger value of $\mathbb{E}[X]$ means the more time for BS keeping in sleep model, so the curve of $\mathbb{E}\left[P_{\text {save }}\right]$ is similar with $\mathbb{E}[X]$. The average value of vehicular speed together with the energy consumption of switching BS between active and sleep mode will affect the average power saved, as reflected in the third part of (20). When the speed of vehicles is higher, the sojourn time in sleep model is less, so leading to less energy saving. Because of the small value of $\int_{D}^{\infty} \frac{1}{x} f_{x}(x) \mathrm{d} x$, the difference between different distributions is nearly negligible as depicted in Fig. 4 While considering 
a ideal condition that the handover cost is zero, the speed distribution will no longer influence the energy saving.

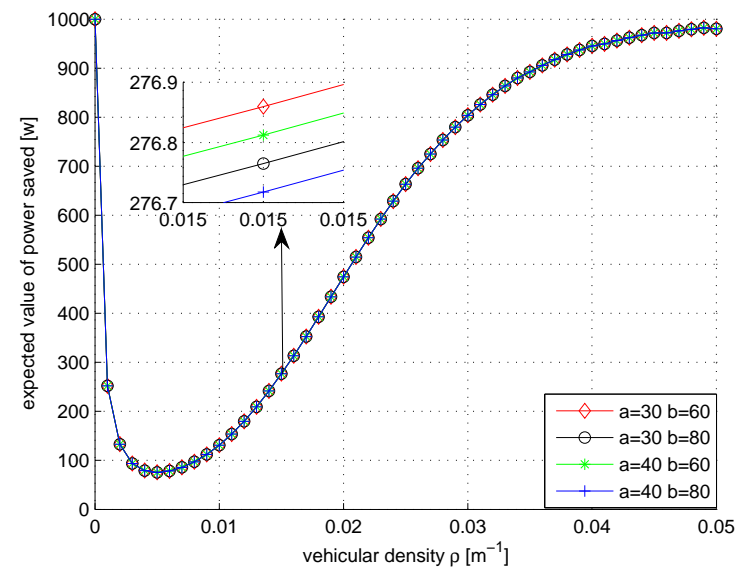

Fig. 4. Average power saved, $r_{0}=200 \mathrm{~m}$.

Since our proposed sleeping schedule takes multi-hop communication into consideration, we wold like to compare our analytical results with the scenario proposed in [11]. As we can see in Fig. 5, our strategy has a better performance especially when the vehicular density is high, in this situation. we can take full advantage of the direct communication between vehicles to save energy of BSs, while in the situation without multi-hop, BSs try to communicate with every vehicles in its coverage, causing a huge amount of energy consumption.

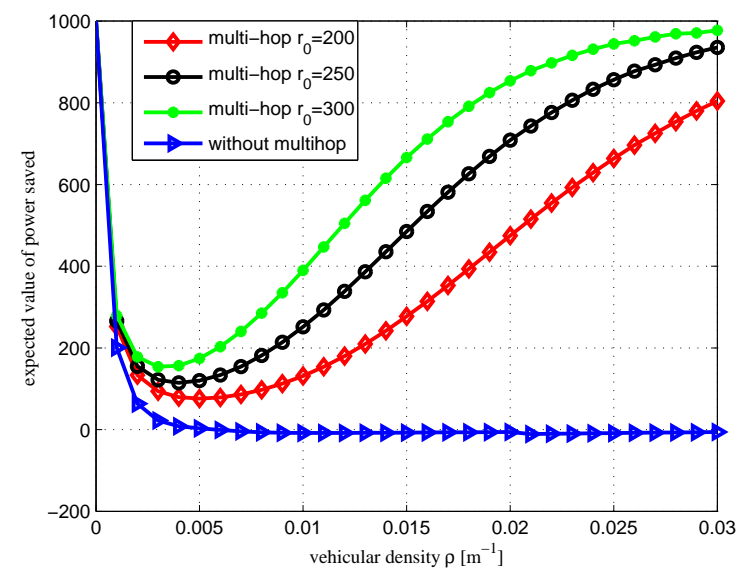

Fig. 5. Our sleeping strategy compares with the scenario without multi-hop communication, where $a=40 \mathrm{~km} / \mathrm{h}, b=80 \mathrm{~km} / \mathrm{h}$.

\section{Vi. CONClusions And Future Work}

This paper considers a multi-hop communication via connections among vehicles, and analyzes the energy efficiency of BS sleeping strategy in 1-D cluster-based vehicular networks. Analytical results have been provided for the expected time interval in sleep mode of a BS. We also investigate the effects caused by network parameters such as vehicular speed distribution. Comparing with the sleeping strategy without considering the multi-hop communication between vehicles, our sleeping schedule has a great improvement in energy saving of BSs.

In our system model, a cluster is formed only by vehicles whose distance with adjacent vehicles is less than $r_{0}$, so the clusters are not stable for vehicular speed changes over time therefore intervals also change, this is not beneficial for information transmission. Then in the future work, we may propose a better ways to form clusters and analyze the energy efficiency, in which two adjacent vehicles in a cluster satisfy that the distance between them is less than a threshold, and their speeds are close enough as well.

\section{REFERENCES}

[1] C. Ren, J. Chen, Y. Kuo, and L. Yang, "Differential successive relaying scheme for fast and reliable data delivery in vehicular ad hoc networks," Communications, IET, vol. 9, no. 8, pp. 1088-1095, 2015.

[2] S. Keykhaie and A. Mahmoudifar, "Study of connectivity in a vehicular ad hoc network with random node speed distribution," in New Technologies, Mobility and Security (NTMS), 2014 6th International Conference on, March 2014, pp. 1-4.

[3] X. Ge, H. Cheng, M. Guizani, and T. Han, "5g wireless backhaul networks: challenges and research advances," Network, IEEE, vol. 28 , no. 6, pp. 6-11, Nov 2014.

[4] J. Wu, Y. Zhang, M. Zukerman, and E.-N. Yung, "Energy-efficient basestations sleep-mode techniques in green cellular networks: A survey," Communications Surveys Tutorials, IEEE, vol. 17, no. 2, pp. 803-826, Secondquarter 2015.

[5] L. Xiang, X. Ge, C.-X. Wang, F. Y. Li, and F. Reichert, "Energy efficiency evaluation of cellular networks based on spatial distributions of traffic load and power consumption," Wireless Communications, IEEE Transactions on, vol. 12, no. 3, pp. 961-973, March 2013.

[6] C. Shao, S. Leng, Y. Zhang, A. Vinel, and M. Jonsson, "Analysis of connectivity probability in platoon-based vehicular ad hoc networks," in Wireless Communications and Mobile Computing Conference (IWCMC), 2014 International, Aug 2014, pp. 706-711.

[7] Z. Zhang, G. Mao, and B. Anderson, "On the information propagation process in mobile vehicular ad hoc networks," Vehicular Technology, IEEE Transactions on, vol. 60, no. 5, pp. 2314-2325, Jun 2011.

[8] S. Mehar, S. Senouci, A. Kies, and M. Zoulikha, "An optimized roadside units (rsu) placement for delay-sensitive applications in vehicular networks," in Consumer Communications and Networking Conference (CCNC), 2015 12th Annual IEEE, Jan 2015, pp. 121-127.

[9] A. Bousia, A. Antonopoulos, L. Alonso, and C. Verikoukis, ""green" distance-aware base station sleeping algorithm in lte-advanced," in Communications (ICC), 2012 IEEE International Conference on, June 2012, pp. 1347-1351.

[10] B. J. Lee, Y. Choi, S. Park, and S. H. Rhee, "An energy efficient power control mechanism for base stations in mobile communication systems," in Information Networking (ICOIN), 2011 International Conference on, Jan 2011, pp. 378-383.

[11] T. Han, Z. Zhang, M. Hu, G. Mao, X. Ge, Q. Li, and L. Wang, "Energy efficiency of cooperative base station sleep scheduling for vehicular networks," in Vehicular Technology Conference (VTC Spring), 2014 IEEE 79th, May 2014, pp. 1-5.

[12] X. Li, B.-J. Hu, H. Chen, B. Li, H. Teng, and M. Cui, "Multi-hop delay reduction for safety-related message broadcasting in vehicle-to-vehicle communications," Communications, IET, vol. 9, no. 3, pp. 404-411, 2015.

[13] G. Mao, Z. Zhang, and B. Anderson, "Probability of k-hop connection under random connection model," Communications Letters, IEEE, vol. 14, no. 11, pp. 1023-1025, November 2010.

[14] A. Bousia, E. Kartsakli, L. Alonso, and C. Verikoukis, "Dynamic energy efficient distance-aware base station switch on/off scheme for lte-advanced," in Global Communications Conference (GLOBECOM), 2012 IEEE, Dec 2012, pp. 1532-1537.

[15] M. Ericson, "Total network base station energy cost vs. deployment," in Vehicular Technology Conference (VTC Spring), 2011 IEEE 73rd, May 2011, pp. 1-5. 